\title{
Swiss Quality Award: effet garanti
}

\author{
Participer au Swiss Quality Award, le jeu en vaut la chandelle - comme I'ont \\ constaté deux lauréats des années 2011 et 2013, le Dr Ueli Grüninger du Collège de \\ médecine de premier recours, et le Dr phil. nat. Priska Vonbach de l'Hôpital des \\ enfants de Zurich. Vous aussi, tentez votre chance et inscrivez votre projet d'ici au
} 30 avril 2014 !*

Interview:

Fabienne Hohl

* Vous trouverez les posters (en allemand seulement) des projets lauréats «Coaching santé» et «kinderdosierungen.ch» dans l'édition en ligne du BMS www.bullmed.ch $\rightarrow$ Numéro actuel et sur www.swissqualitaward.ch $\rightarrow$ Les projets.
Correspondance:

Michelle Gerber

FMH / Division DDQ

Elfenstrasse 18

CH-3000 Berne

Tél. 0313591111

Fax 0313591112

info[at]swissqualityaward.ch www.swissqualityaward.ch

Dr Ueli Grüninger Collège de médecine de premier recours (CMPR)

Landhausweg 26

CH-3007 Berne

Tél. 0313700671

ueli.grueninger[at]hin.ch www.gesundheitscoachingkhm.ch

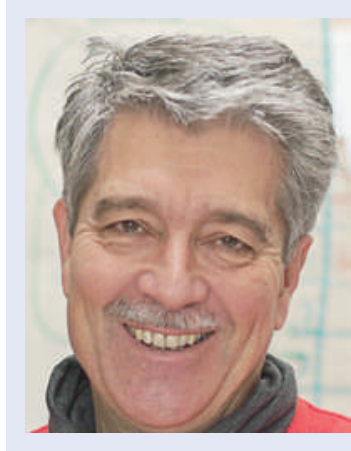

Dr Ueli Grüninger, directeur du Collège de médecine de premier recours: «Ce prix signifie que la question de la collaboration entre patient et médecin a fait son chemin également dans le domaine de la gestion de la qualité.»

Dr Grüninger, en 2011, vous et votre équipe du Collège de médecine de premier recours avez remporté le Swiss Quality Award avec votre projet "Coaching santé». Que signifie ce prix pour vous?

Ueli Grüninger: Ce prix a été une grande surprise pour nous. Cela signifiait que contrairement à ce que nous pensions, la question de la collaboration entre patient et médecin avait fait son chemin également dans le domaine de la gestion de la qualité. La reconnaissance du public mais aussi la légitimation de nos pairs nous ont renforcés dans notre idée d'être sur la bonne voie et nous ont fortement incités à étendre notre projet à l'ensemble du pays.

Comment le coaching santé a-t-il évolué depuis la remise du Swiss Quality Award?

Nous avons poursuivi activement notre travail afin d'évaluer le projet-pilote, qui a réuni quelque 20 cabinets médicaux et 1045 patients, et de publier les résultats très prometteurs que nous avons obtenus [1]. A titre d'exemple: 91\% des patients approchés ont rejoint le projet, et 37\% sont allés jusqu'au bout, soit trois fois plus qu'attendu. La moitié de ces patients sont parvenus à changer leur comportement, ce qui est un résultat remarquable en comparaison avec d'autres interventions médicales. La praticabilité du coaching a été jugée très bonne à la fois par les patients et les médecins, et le projet a fait l'objet d'une large acceptation de part et d'autre. Forts de ce succès, nous prévoyons désormais d'élargir ce projet à toute la Suisse. Nous menons actuellement des dis- cussions avec les décideurs politiques et les institutions spécialisées afin de garantir le financement du projet mais aussi de négocier des conditions favorables, dont la rémunération adéquate du conseil médical et à long terme, l'intégration du coaching santé dans la formation des professionnels de la santé.

\section{L'intérêt pour votre projet a-t-il augmenté depuis la remise du prix?}

La demande pour notre projet est en constante augmentation, ce qui nous réjouit. Nous organisons d'ores et déjà des formations continues dans les cantons d'Argovie et de Saint-Gall ainsi qu'au Liechtenstein, en collaboration avec les départements de la santé et les sociétés médicales. D'autres formations sont en préparation. La formation continue offre quatre modules: cours de sensibilisation (2-3h), atelier d'une journée avec des patients standardisés/acteurs, rencontres de suivi et apprentissage personnel en ligne.

\section{Le prix vous a-t-il permis de nouer des contacts utiles?}

La visibilité et la crédibilité obtenues grâce au prix sont un soutien précieux dans notre recherche de partenaires et de sponsors en vue d'étendre le programme de coaching santé à l'ensemble du pays. Cela nous a également permis d'approcher des groupes cibles autres que les médecins installés, à savoir des médecins en formation postgraduée, des étudiants en médecine ou d'autres professionnels de la santé, par ex. des assistantes médicales.

Les démarches en faveur de la qualité des soins bénéficient d'un large ancrage en Suisse. Le Swiss Quality Award a-t-il encore son utilité?

Ce prix est important dans le sens où il permet de montrer l'extraordinaire diversité des activités en faveur de la qualité des soins. Mais la visibilité n'est pas tout. C'est pourquoi je souhaiterais que le Swiss Quality Award contribue aussi à ce que l'on place notre confiance moins dans la technologie mais davantage dans le système et les possibilités qu'il offre ainsi que dans l'interaction. 


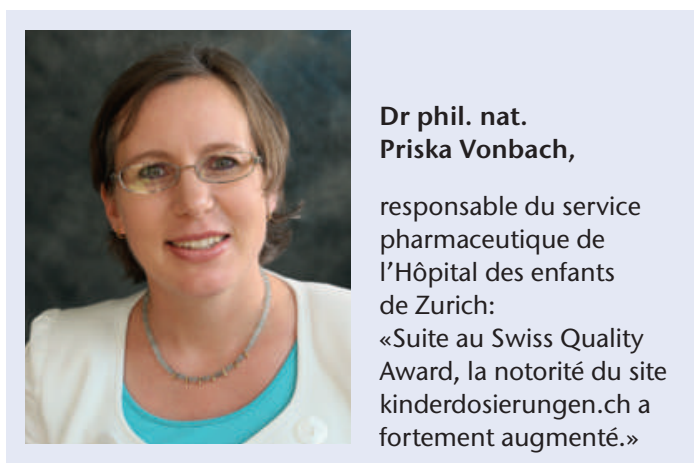

Dr Vonbach, en 2013, l'équipe de l'Hôpital des enfants de Zurich a remporté le Swiss Quality Award pour son nouveau site internet kinderdosierungen.ch. Qu'est-ce qui a convaincu le jury?

Priska Vonbach: Alliant innovation et qualité, notre site internet kinderdosierungen.ch était prédestiné pour le Swiss Quality Award. «Innovation», parce qu'à notre connaissance, il n'existe aucune autre banque de données publique sur les dosages pédiatriques qui permette également de calculer les dosages individuels - et «qualité», parce que la sécurité des patients était depuis le début clairement au centre du projet et qu'elle reste notre objectif premier.

Participer à un concours nécessite beaucoup de ressources cet investissement a-t-il porté ses fruits?

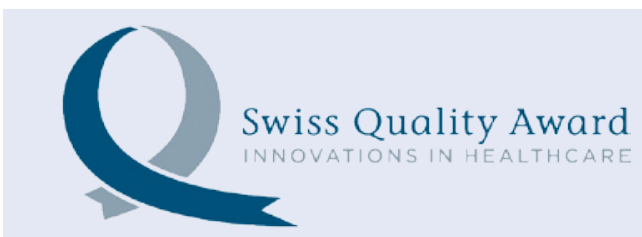

Swiss Quality Award: inscrivez-vous maintenant! Les innovations dans le domaine de la gestion de la qualité bénéficient non seulement aux patients mais aussi à l'ensemble du secteur de la santé. C'est pourquoi le Swiss Quality Award récompense les meilleurs projets en faveur de la qualité dans trois catégories, dotées chacune de 10000 francs: «secteur ambulatoire», «secteur hospitalier» et «projet intersectoriel». A partir de cette année, le jury décernera également une récompense d'une valeur de $\mathbf{2 0 0 0}$ francs pour le meilleur poster, le «Swiss Quality Poster-Award». Les trois organismes responsables que sont la Fédération des médecins suisses (FMH), l'Institut de recherche évaluative en médecine (IEFM) de I'Université de Berne et la Société suisse pour le management de qualité dans la santé (SQMH) se réjouissent d'ores et déjà de découvrir votre projet; n'hésitez donc pas à participer au concours! Les inscriptions sont ouvertes jusqu'au 30 avril 2014. Pour de plus amples informations: www. swissqualityaward.ch.
Les ressources mobilisées pour le concours sont restées tout à fait raisonnables. Naturellement, en tant que lauréats, l'investissement a porté ses fruits. La notoriété de www.kinderdosierungen.ch a fortement augmenté, comme le montrent les statistiques du site. De plus, le logo du Swiss Quality Award facilite grandement la recherche de sponsors.

Comment a évolué le site kinderdosierungen.ch depuis la remise du Swiss Quality Award?

Depuis décembre 2013, toutes les données sont également accessibles en français sous www.posologiespediatriques.ch. Par ailleurs, nous pouvons désormais intégrer les médicaments génériques dans notre banque de données et nous prévoyons donc de l'élargir de manière importante durant le premier trimestre 2014, en plus de la mise à jour usuelle. Nous sommes également en train de programmer une version adaptée aux smartphones et tablettes. Vu que les utilisateurs de l'Hôpital des enfants de Zurich devront désormais renoncer à une version imprimée de nos dosages, nous leur devons bien cela. Enfin, nous travaillons avec d'autres cliniques pour enfants ainsi qu'avec l'OFSP sur un projet-pilote visant à harmoniser les dosages.

Jetons un regard vers l'avenir: selon vous, quelle orientation le Swiss Quality Award va-t-il prendre ces prochaines années?

J'attends des innovations qu'elles s'attachent à répondre aux défis qui nous attendent. Dans ce contexte, le thème de la cybersanté sera l'une de nos principales préoccupations, de même que la coordination de la prise en charge des patients entre secteurs stationnaire et ambulatoire, qui revêt encore plus d'importance depuis l'introduction de SwissDRG. J'attends également des innovations qu'elles permettent d'actualiser nos connaissances médicales et d'augmenter l'attrait de l'hôpital en tant que lieu de travail, afin de contrer la pénurie qui s'annonce.

Selon vous, qu'est-ce qui caractérise une bonne démarche en faveur de la qualité?

Pour moi, la sécurité des patients est primordiale, car c'est un aspect auquel on attache malheureusement encore trop peu d'importance, les conséquences d'une mauvaise prise en charge étant bien souvent sous-estimées. De manière générale, que ce soit par des thérapies plus efficaces, une meilleure sécurité ou des traitements plus efficients - comprenez: moins chers -, la qualité des soins doit bénéficier avant tout aux patients.

1 Neuner-Jehle S, Schmid M, Grüninger U. The «Health Coaching» programme: a new patient-centred and visually supported approach for health behaviour change in primary care. BMC Family Practice. 2013; 14(100). www.biomedcentral.com/1471-2296/14/100. 